\title{
A variational approach to a BVP arising in the modelling of electrically conducting solids
}

\author{
Suheil A. Khuri ${ }^{1 *}$, Abdul-Majid Wazwaz ${ }^{2}$ \\ 1 Department of Mathematics and Statistics, American University of Sharjah - UAE \\ 2 Department of Mathematics, Saint Xavier University, Chicago, IL 60655 - USA
}

Received 18 August 2012; accepted 27 August 2012

\begin{abstract}
The purpose of this paper is to apply an amended variational scheme, based on an adapted domain decomposition, for the solution of a second-order nonlinear boundary value problem that arises in applications involving the diffusion of heat generated by positive temperature dependent sources. The underlying idea of this approach is to decompose the original interval prior to the implementation of the iterative variational approach in order to improve the accuracy and acquire uniform convergence to the exact solution over the entire domain. Convergence analysis is given and then numerical experiments are carried out to make obvious the convergence, accuracy and efficient applicability of the method.

Keywords: Variational iterative method $\cdot$ Second-order nonlinear boundary value problem $\cdot$ Domain decomposition

(C) Versita sp. z o.o.
\end{abstract}

\section{Introduction}

The purpose of this article is to apply a novel version of the variational iterative scheme (VIM) for the solution of the following second-order nonlinear boundary value problem:

$$
y^{\prime \prime}(t)=\mu(t) e^{\rho(t) y(t)}
$$

defined on the interval $[0,1]$ and subject to the following boundary conditions:

$$
y(0)=0, \quad y(1)=0
$$

Here $\rho(t)$ and $\mu(t)$ are continuous functions of $t$. This problem arises in the modelling of electrically conducting

*E-mail: email@server.domain solids. For instance, if $\rho=1$ it arises in the analysis of Joule losses in electrically conducting solids where $\mu$ represents the square of the constant current and $e^{y}$ the temperature-dependent resistance, or in frictional heating with $\mu$ representing the square of the constant shear stress and $e^{y}$ the temperature-dependent fluidity.

In recent years we witnessed increased research interest in this type of problem, which is a generalized form of Bratu's problem. Agarwal and Loi [1] considered the $n$ th-order nonlinear differential equation

$$
y^{(n)}(t)=f\left(t, y(t), y^{\prime}(t), \ldots, y^{(n-1)}(t)\right)
$$

with multipoint boundary conditions and proved existence and uniqueness via application of Picard's iterative method. In particular, as an illustration of a specific case, the authors considered the two-point boundary value problem, 
that occurs in diffusion theory [2], given by

$$
\begin{aligned}
& y^{\prime \prime}(t)=\mu e^{\rho y(t)} \\
& y(0)=y(1)=0,
\end{aligned}
$$

where $\mu$ and $\rho$ are constants. They obtained explicit solutions and discussed their qualitative properties. Goyal [6] studied an extended form of this problem, namely,

$$
\begin{aligned}
& y^{\prime \prime}(t)=\mu(t) e^{\rho y(t)}, \\
& y(0)=y(1)=0,
\end{aligned}
$$

and attained explicit solutions for constant $\rho>0$ and $\mu(t)>0$ satisfying the condition $\frac{d^{2}}{d t^{2}}(\ln \lambda(t))=0$.

Most recently, Bougoffa [3] and Misir and Tiryaki [13] investigated the more general problem (1)-(2). In both papers, the authors derived conditions for the existence of nontrivial solutions; the methods in both are similar, except the author in [3] has modified the transformation used in [13]. Finally, it is important to point out that the special case, well-known as Bratu's problem,

$$
\begin{aligned}
& y^{\prime \prime}(t)=\mu e^{y(t)} \\
& y(0)=y(1)=0
\end{aligned}
$$

where $\mu$ is a constant, has been extensively studied in the literature and this is authenticated by the spectrum of articles. In higher dimensions, Bratu's problem models a combustion problem in a slab. Indeed, it has been a popular testbed for numerical and perturbation methods. For instance, lately Jalilian [9] developed smooth approximate solutions of the one-dimensional Bratu's problems by using nonpolynomial spline function. Boyd [4] introduced two methods for simultaneously approximating both branches of a two-branched function using Chebyshev polynomials and were applied to Bratu's problem. In a separate paper, Boyd [5] applied very low order spectral methods to generate accurate, explicit, analytic approximations of the Bratu function and showed that it is so well approximated by a parabola. Some theoretical gaps that still exist are filled for this heavily-studied problem. Khuri [10] introduced a Laplace transform decomposition numerical algorithm for solving Bratu's problem. Numerical and perturbation solutions of this problem were obtained by numerous numerical methods, see [5], [9] and [10] and the references therein. The core goal of this article is to implement a tailored method, based on domain decomposition and the variational iterative method (VIM), for the numerical solution of the second-order nonlinear boundary value problem (1)-(2). The VIM, which typically yields series solution, converges pretty fast near the initial point $x=0$ and is highly accurate using few iterates but worsens away from the origin, namely as $x$ becomes larger and larger. To overcome this setback, the domain will be decomposed in such a way to achieve uniform convergence over the entire domain and the VIM is applied repeatedly over the subdomains.

Lately, there has been a prevalent interest (see $[7,8]$, $[11,12]$ and $[14]$ and the references therein) in the VIM as a rather prominent scheme for attaining exact and/or accurate numerical solutions of a variety of problems such as algebraic, differential, partial-differential, functionaldelay and integro-differential equations. The main thrust of the method is based on constructing a correction functional, using a general Lagrange multiplier selected in a certain fashion, that its correction solution is improved with respect to the initial approximation or to the trial function. The method results in a solution in the form of a convergent successive approximations that may give or converge to the exact solution. The approach is reliable and efficient and handles the equations without imposing limiting assumptions that may alter the physical structure of the solutions.

The balance of this paper is organized as follows. In Section 2, the domain decomposition variational approach is presented for the numerical solution of the second-order nonlinear boundary value problem. In Section 3, numerical results are given to confirm the accuracy of the technique. Finally, a discussion is given that briefly summarizes the results of the paper's content.

\section{Numerical Method}

In this section, we present the variational iterative scheme as well as the domain decomposition strategy.

\subsection{Variational Scheme}

We will illustrate the basic concepts of VIM, in constructing the correction functional, as it applies to problem (1)-(2). According to VIM, we construct the following correction functional:

$$
y_{n+1}(x)=y_{n}(x)+\int_{0}^{x} \lambda(s)\left[\left(y_{n}\right)_{s s}-\mu(s) e^{\rho(s) \tilde{y}_{n}}\right] d s,
$$

where the subscript $n=0,1,2, \ldots$ denotes the $n$th order approximation. Further, $\lambda$ is a general Lagrangian multiplier that can be identified optimally via the variational theory and $\tilde{y}_{n}$ is regarded as a restricted variation (that is, $\left.\delta\left(\tilde{y}_{n}\right)=0\right)$.

To find the optimal value of $\lambda(s)$, we advance in the following manner: first, we apply the variation with respect to $y_{n}$ on both sides of (3). Thus 


$$
\frac{\delta y_{n+1}}{\delta y_{n}}=1+\frac{\delta}{\delta y_{n}}\left(\int_{0}^{x} \lambda(s)\left[\left(y_{n}\right)_{s s}-\mu(s) e^{\rho(s) \tilde{y}_{n}}\right] d s\right)
$$

or equivalently

$$
\begin{aligned}
\delta y_{n+1}(x)= & \delta y_{n}(x) \\
& +\delta\left(\int_{0}^{x} \lambda(s)\left[\left(y_{n}\right)_{s s}-\mu(s) e^{\rho(s) \tilde{y}_{n}}\right] d s\right)
\end{aligned}
$$

The second term in the integrand drops out upon taking the variation. Consequently we have

$$
\delta y_{n+1}(x)=\delta y_{n}(x)+\delta\left(\int_{0}^{x} \lambda(s)\left(y_{n}\right)_{s s} d s\right)
$$

Integrating by parts results

$$
\begin{aligned}
\delta y_{n+1}(x)= & \delta y_{n}(x)+\delta\left[\lambda(x)\left(y_{n}\right)_{s}(x)\right] \\
& -\delta\left(\int_{0}^{x} \lambda^{\prime}(s)\left(y_{n}\right)_{s} d s\right) .
\end{aligned}
$$

A second integration by parts gives

$$
\begin{aligned}
\delta y_{n+1}(x)= & \delta y_{n}(x)+\delta\left[\lambda(x)\left(y_{n}\right)_{s}(x)\right]-\delta\left[\lambda^{\prime}(x) y_{n}(x)\right] \\
& +\delta\left(\int_{0}^{x} \lambda^{\prime \prime}(s) y_{n}(s) d s\right),
\end{aligned}
$$

which can be written as

$$
\begin{aligned}
\delta y_{n+1}(x)= & {\left[1-\lambda^{\prime}(x)\right] \delta y_{n}(x)+\delta\left[\lambda(x)\left(y_{n}\right)_{s}(x)\right] } \\
& +\delta\left(\int_{0}^{x} \lambda^{\prime \prime}(s) y_{n}(s) d s\right) .
\end{aligned}
$$

Hence, we obtain the stationary conditions:

$$
\begin{aligned}
& 1-\left.\lambda^{\prime}(s)\right|_{s=x}=0, \\
& \left.\lambda(s)\right|_{s=x}=0, \\
& \lambda^{\prime \prime}(s)=0 .
\end{aligned}
$$

Upon solving equations (10), the Lagrange multipliers are identified as

$$
\lambda(s)=s-x .
$$

The variational iterative procedure is thus given by

$$
y_{n+1}(x)=y_{n}(x)+\int_{0}^{x}(s-x)\left[\left(y_{n}\right)_{s s}-\mu(s) e^{\rho(s) y_{n}(s)}\right] d s .
$$

\subsection{Domain decomposition algorithm}

The (VIM) yields a series solution about the starting point, in this case $x=0$, and therefore the approximation is very accurate close to zero and worsens away from it. To overcome this numerical deficiency we will propose a domain decomposition that will improve the accuracy away from the origin. More specifically, the applicable domain $[0,1]$ is decomposed at first into two subintervals $[0,1]=\left[0, \delta_{1}\right] \cup\left[\delta_{1}, 1\right]$ that are non-overlapping except at the mesh point $x=\delta_{1}$. Then the (VIM) is applied on the first subinterval $\left[0, \delta_{1}\right]$ and $\delta_{1}$ is chosen in such a way till the error of the approximation is small enough and is within a prescribed tolerance as follows:

$$
\left|y_{n_{1}}\left(\delta_{1}\right)-y_{n_{1}-1}\left(\delta_{1}\right)\right|<T o l,
$$

where $y_{n_{1}}\left(\delta_{1}\right)$ is the numerical approximation obtained by the first application of the (VIM), Tol is the tolerance and $n_{1}$ denotes the number of (VIM) iterates that are needed to satisfy the latter condition. We start with the initial approximation $y_{0}=y(0)+y^{\prime}(0) x$. From the boundary conditions (2), we have $y_{0}=b x$ where the constant $b$ is determined from the resulting series solution upon imposing the boundary condition $y(1)=0$.

Upon the determination of $\delta_{1}$, the initial condition is estimated at $x=\delta_{1}$, that is, the value of $y\left(\delta_{1}\right)$. Then the (VIM) is applied again on the second subdomain $\left[\delta_{1}, 1\right]$ where the iterative scheme becomes as follows:

$$
y_{n+1}(x)=y_{n}(x)+\int_{\delta_{1}}^{x} \lambda(s)\left[\left(y_{n}\right)_{s s}-\mu(s) e^{\rho(s) \tilde{y}_{n}}\right] d s,
$$

where the new initial conditions are:

$$
y\left(\delta_{1}\right)=y_{n_{1}}\left(\delta_{1}\right), \quad y(1)=1
$$

. The initial approximation is now taken to be

$$
y_{0}=y\left(\delta_{1}\right)+y^{\prime}\left(\delta_{1}\right)\left(x-\delta_{1}\right)=y\left(\delta_{1}\right)+k\left(x-\delta_{1}\right),
$$

where the constant $k$ is found from the boundary condition $y(1)=0$. Again, this interval is split into two subdomains $\left[\delta_{1}, 1\right]=\left[\delta_{1}, \delta_{2}\right] \cup\left[\delta_{2}, 1\right]$ and the (VIM) is implemented and $\delta_{2}$ is estimated in a similar fashion. This algorithm is repeated to calculate the $\delta_{3}, \delta_{4} \ldots$ till uniform convergence is achieved.

\section{Convergence analysis}

According to (3), the VIM iteration formulation is given by:

$$
y_{n+1}(x)=y_{n}(x)+\int_{0}^{x} \lambda(s)\left[\left(y_{n}\right)_{s s}-\mu(s) e^{\rho(s) y_{n}(s)}\right] d s,(13)
$$

where $n \geq 0$. 


\section{Theorem 1.}

The sequences $\left\{y_{n}(x)\right\}_{n=1}^{\infty}$, where $x \in[0,1]$, defined by (13) with $y_{0}(x)=b x$ ( $b$ real constant) converges to the exact solution, $y(x)$, of problem (1)-(2).

Proof. The iterative scheme (3) can be rewritten as

$$
\begin{aligned}
y_{n+1}(x)-y(x)= & y_{n}(x)-y(x)+\int_{0}^{x} \lambda(s)\left[\left(y_{n}-y\right)_{s s}\right. \\
& \left.+y_{s s}-\mu(s) e^{\rho(s) y_{n}(s)}\right] d s .
\end{aligned}
$$

Note that the integrand was expressed in terms of $y_{n}-y$ and $y$ was subtracted from both sides of the equation. Since $y(x)$ is the true solution of (1)-(2), thus the term $y_{s s}$ can be replaced by $\mu(s) e^{\rho(s) y(s)}$.

Setting $E_{n}(x)=y_{n}(x)-y(x)$, equation (14) becomes

$$
\begin{aligned}
E_{n+1}(x)= & E_{n}(x)+\int_{0}^{x} \lambda(s)\left[\left(E_{n}(s)\right)_{s s}\right. \\
& \left.+\mu(s) e^{\rho(s) y(s)}-\mu(s) e^{\rho(s) y_{n}(s)}\right] d s .
\end{aligned}
$$

Integrating the first term in the integrand twice by parts we obtain

$$
\begin{aligned}
E_{n+1}(x)= & E_{n}(x)+\lambda(x)\left(E_{n}\right)_{s}(x)-\lambda^{\prime}(x) E_{n}(x) \\
& +\int_{0}^{x} \lambda^{\prime \prime}(s) E_{n}(s) d s \\
& +\int_{0}^{x} \lambda(s) \mu(s)\left[e^{\rho(s) y(s)}-e^{\rho(s) y_{n}(s)}\right] d s .
\end{aligned}
$$

Substituting the three stationary conditions in (10) into the last equation we get

$$
E_{n+1}(x)=\int_{0}^{x} \lambda(s) \mu(s)\left[e^{\rho(s) y(s)}-e^{\rho(s) y_{n}(s)}\right] d s
$$

Operating with the $L^{2}$-norm on both sides we have the following inequality.

$$
\begin{aligned}
\left\|E_{n+1}(x)\right\|_{L^{2}} \leq & \int_{0}^{x}\|\lambda(s)\|_{L^{2}}\|\mu(s)\|_{L^{2}} \| e^{\rho(s) y(s)} \\
& -e^{\rho(s) y_{n}(s)} \|_{L^{2}} d s \\
\leq & \|\lambda(s)\|_{\infty}\|\mu(s)\|_{\infty} \int_{0}^{x} \| e^{\rho(s) y(s)} \\
& -e^{\rho(s) y_{n}(s)} \|_{L^{2}} d s
\end{aligned}
$$

where $\|\lambda(s)\|_{\infty}=\max _{s \in[0,1]}|\lambda(s)|$ and $\|\mu(s)\|_{\infty}=\max _{s \in[0,1]}|\mu(s)|$. Note we assumed that the function $\mu(s)$ is continuous hence bounded on the specified interval. The fact that $\lambda(s)$ is also bounded follows from equation (11), that is $\lambda(s)=(x-s)$, since this means that

$$
\|\lambda(s)\|_{\infty}=\|x-s\|_{\infty} \leq\|x\|_{\infty}+\|s\|_{\infty} \leq 1+1=2 .
$$

By the Mean Value Theorem applied to the function $f(\rho, y)$, assuming that $f_{y}$ exists and is bounded, we have

$$
f\left(\rho, y_{2}\right)-f\left(\rho, y_{1}\right)=f_{y}(\rho, \xi)\left(y_{2}-y_{1}\right)
$$

Here $\xi$ is the intermediate point between $y_{1}$ and $y_{2}$. Therefore

$$
\begin{aligned}
\left\|f\left(\rho, y_{2}\right)-f\left(\rho, y_{1}\right)\right\|_{L^{2}} & =\left\|f_{y}(\rho, \xi)\right\|_{L^{2}}\left\|y_{2}-y_{1}\right\|_{L^{2}} \\
& \leq\left\|f_{y}(\rho, \xi)\right\|_{\infty}\left\|y_{2}-y_{1}\right\|_{L^{2}}
\end{aligned}
$$

In particular, since the solution of Bratu's problem has been proven to be bounded (see $[4,5,9,10]$ and the references therein), therefore we have

$$
\begin{aligned}
\left\|e^{\rho(s) y_{n}(s)}-e^{\rho(s) y(s)}\right\|_{L^{2}} & \leq\left\|\rho(s) e^{\rho(s) \xi(s)}\right\|_{\infty}\left\|y_{n}-y\right\|_{L^{2}} \\
& =\left\|\rho(s) e^{\rho(s) \xi(s)}\right\|_{\infty}\left\|E_{n}(s)\right\|_{L^{2}} .
\end{aligned}
$$

It follows from equations (21) and (20) that

$$
\begin{aligned}
&\left\|E_{n+1}(x)\right\|_{L^{2}} \leq\|\lambda(s)\|_{\infty}\|\mu(s)\|_{\infty} \int_{0}^{x} \| e^{\rho(s) y(s)} \\
&-e^{\rho(s) y_{n}(s)} \|_{L^{2}} d s \\
& \leq \lambda(s)_{\infty} \mu(s)_{\infty}\left\|\rho(s) e^{\rho(s) \xi(s)}\right\|_{\infty} \int_{0}^{x}\left\|E_{n}(s)\right\|_{L^{2}} d s \\
& \leq \lambda(s)_{\infty} \mu(s)_{\infty} \rho(s)_{\infty} e^{\mu(s)_{\infty} \xi(s)_{\infty}} \int_{0}^{x}\left\|E_{n}(s)\right\|_{L^{2}} d s .
\end{aligned}
$$

Let

$$
\begin{aligned}
& L=\max _{s \in[0,1]}|\lambda(s)|, \quad M=\max _{s \in[0,1]}|\mu(s)|, \quad N=\max _{s \in[0,1]}|\rho(s)|, \\
& \text { and } P=\max _{s \in[0,1]}|\xi(s)| .
\end{aligned}
$$

Then, from inequality (21) we get

$$
\left\|E_{n+1}(x)\right\|_{L^{2}} \leq L M N e^{M P} \int_{0}^{x}\left\|E_{n}(s)\right\|_{L^{2}} d s .
$$

Proceeding by induction and setting the constant $L_{1}=$ $L M N e^{M P}$ we obtain 


$$
\begin{aligned}
& \left\|E_{1}(x)\right\|_{L^{2}} \leq L_{1} \int_{0}^{x}\left\|E_{0}(s)\right\|_{L^{2}} d s \leq L_{1}\left\|E_{0}(s)\right\|_{\infty} \int_{0}^{x} d s=L_{1}\left\|E_{0}(s)\right\|_{\infty} x, \\
& \left\|E_{2}(x)\right\|_{L^{2}} \leq L_{1} \int_{0}^{x}\left\|E_{1}(s)\right\|_{L^{2}} d s \leq L_{1}^{2}\left\|E_{0}(s)\right\|_{\infty} \int_{0}^{x} s d s=L_{1}^{2}\left\|E_{0}(s)\right\|_{\infty} \frac{x^{2}}{2}, \\
& \ldots \\
& \left\|E_{n+1}(x)\right\|_{L^{2}} \leq L_{1} \int_{0}^{x}\left\|E_{n}(s)\right\|_{L^{2}} d s \leq L_{1}^{n+1}\left\|E_{0}(s)\right\|_{\infty} \int_{0}^{x} \frac{s^{n}}{n !} d s=L_{1}^{n+1}\left\|E_{0}(s)\right\|_{\infty} \frac{x^{n+1}}{(n+1) !} .
\end{aligned}
$$

Here $\left\|E_{0}(s)\right\|_{\infty}=\max _{s \in[0,1]}\left|E_{0}(s)\right|$

Since $\left\|E_{0}(s)\right\|_{\infty}=\left\|y_{0}(x)-y(x)\right\|_{\infty}=\|b x-y(x)\|_{\infty}$ and $x \in[0,1]$, thus

$$
\begin{aligned}
\left\|E_{0}(s)\right\|_{\infty} & \leq\|b x\|_{\infty}+\|y(x)\|_{\infty}=|b|+\|y(x)\|_{\infty} \\
& =|b|+\max _{x \in[0,1]}|y(x)| .
\end{aligned}
$$

Note that $y(x)$ belongs to $C^{2}[0,1]$ since it is the exact solution of equation (1) hence it is bounded. Letting $c=\max _{x \in[0,1]}|y(x)|$ we have from $(23)$

$$
E_{n+1}(x)_{L^{2}} \leq L_{1}^{n+1}(b+c) \frac{x^{n+1}}{(n+1) !}
$$

Note that for $x \in[0,1]$ the sequence $\left\{\frac{\left(L_{1} x\right)^{n+1}}{(n+1) !}\right\}$ converges uniformly to 0 as $n$ tends to infinity. Hence by (25) it follows that $E_{n+1}(x)_{L^{2}}$ converges to 0 which means $y_{n}(x)$ converges uniformly to $y(x)$.

\section{Numerical examples}

In this section, the variational iterative method (VIM) as well as the mixed domain decomposition variational approach (DDVIM) are implemented for tackling two special cases of Bratu's problem. The first is when $\lambda=1$, the VIM converges to one of the two solutions. In Table 1, the exact solution, for the case $\lambda=1$, is compared with the numerical solution obtained by (VIM). Note that the maximum absolute error is $1.2 \times 10^{-9}$ using only seven iterates. It is worth mentioning that the integral in the (VIM) procedure (12) was handled numerically using Taylor's expansion, that is, the function $e^{\rho(s)}$ in the integrand was replaced and estimated using the first 24 of Maclaurin series expansion. The extreme small absolute maximum error illustrates the accuracy of the method using only few steps of the iterative strategy. The solution is shown in Figure 1.

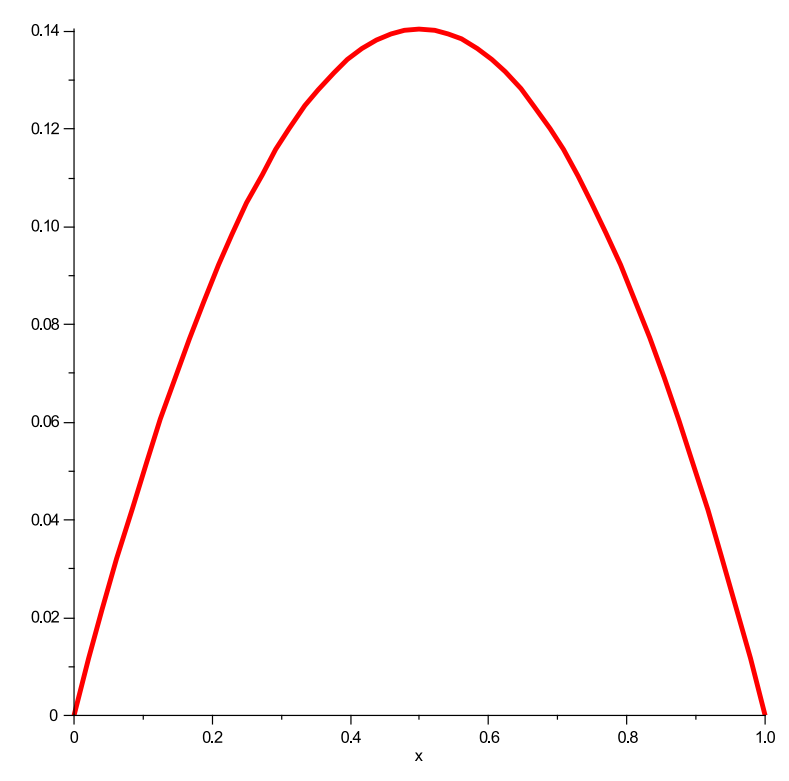

Figure 1. Numerical solution of Bratu's problem for the case $\lambda=1$.

Table 1. (VIM) applied to Bratu's problem for the case $\lambda=1$.

\begin{tabular}{ccc}
\hline $\mathrm{x}$ & Exact Solution & (VIM) Solution \\
\hline \hline 0.1 & 0.0498467900 & 0.0498467912 \\
0.2 & 0.0891899350 & 0.0891899346 \\
0.3 & 0.1176090956 & 0.1176090958 \\
0.4 & 0.1347902526 & 0.1347902539 \\
0.5 & 0.1405392142 & 0.1405392144 \\
0.6 & 0.1347902526 & 0.1347902539 \\
0.7 & 0.1176090956 & 0.1176090958 \\
0.8 & 0.0891899350 & 0.0891899346 \\
0.9 & 0.0498467900 & 0.0498467912 \\
\hline
\end{tabular}

The results for the case $\lambda=2$ are represented in Table 2 . The maximum absolute error for this case is $2.3 \times 10^{-7}$ which is lower than the one obtained for the case $\lambda=1$ if same number of iterates, namely seven, are used. It was observed from experimental data for various values of $\lambda$, besides the case $\lambda=2$, that as the value of $\lambda$ increases more iterates of the (VIM) are needed to maintain a specified level of accuracy. 
Table 2. (VIM) applied to Bratu's problem for the case $\lambda=2$.

\begin{tabular}{ccc}
\hline$x$ & Exact Solution & (VIM) Solution \\
\hline \hline 0.1 & 0.1144107440 & 0.1144106916 \\
0.2 & 0.2064191156 & 0.2064190142 \\
0.3 & 0.2738793116 & 0.2738791616 \\
0.4 & 0.3150893646 & 0.3150891699 \\
0.5 & 0.3289524214 & 0.3289521883 \\
0.6 & 0.3150893646 & 0.3150890988 \\
0.7 & 0.2738793116 & 0.2738790214 \\
0.8 & 0.2064191156 & 0.2064188086 \\
0.9 & 0.1144107440 & 0.1144104331 \\
\hline
\end{tabular}

Table 3. Comparison between (VIM) and (DDVIM) when applied to Bratu's problem for the case $\lambda=1$.

\begin{tabular}{cccc}
\hline$x$ & Exact Solution & (VIM) Solution & (DDVIM) Solution \\
\hline \hline 0.1 & 0.0498467900 & 0.0495163885 & 0.0495163885 \\
0.2 & 0.0891899350 & 0.0885326582 & 0.0885326582 \\
0.3 & 0.1176090956 & 0.1166327018 & 0.1166327018 \\
0.4 & 0.1347902526 & 0.1335092699 & 0.1339156186 \\
0.5 & 0.1405392142 & 0.1389821617 & 0.1397763445 \\
0.6 & 0.1347902526 & 0.1330156072 & 0.1341482492 \\
0.7 & 0.1176090956 & 0.1157348391 & 0.1170972421 \\
0.8 & 0.0891899350 & 0.0874418571 & 0.0888208529 \\
0.9 & 0.0498467900 & 0.0486303817 & 0.0496423649 \\
\hline
\end{tabular}

In Table 3 we demonstrate the numerical results obtained by applying both the (VIM) and (DDVIM) for the case $\lambda=1$. The integral in the procedure (12) was computed numerically using five terms of the Maclaurin series expansion of $e^{\rho(s)}$. More terms of the Taylor's expansion are needed to accurately estimate the integral and obtain better results. However, our main intention in this experiment is to contrast the (VIM) and (DDVIM) strategies. The domain $[0,1]$ was decomposed only into two subdomains $[0, \delta] \cup[\delta, 1]$, where $\delta$ was taken to be 0.3 . The (VIM) was implemented and the initial condition at $\delta$ was found to be $u(\delta)=u(0.3) \approx 0.22939308154999$. The $(\mathrm{VIM})$ is then applied on the subdomain $[0.3,1]$. The iterative formulation (12) on the second subdomain becomes

$$
y_{n+1}(x)=y_{n}(x)+\int_{\delta}^{x}(s-x)\left[\left(y_{n}\right)_{s s}-\mu(s) e^{\rho(s) y_{n}(s)}\right] d s,
$$

with boundary conditions

$$
u(\delta)=0.22939308154999, \quad u(1)=0,
$$

where $\delta=0.3$. Note from the Table that the numerical results improved when applying the DDVIM. More subdivisions are required to attain better accuracy and even piecewise uniform convergence.

\section{Discussion}

A combination of the variational iteration method and the domain decomposition concept is employed to handle the second-order nonlinear boundary value problem. To achieve uniform convergence over the entire domain, we decompose the domain into subdomains where the VIM is used repeatedly. The convergence of the scheme was formally proved. The numerical examples that we examined demonstrate enhancements over existing techniques.

\section{References}

[1] Agarwal R.P., Loi S.L., On approximate Picards iterates for multipoint boundary value problems, Nonlinear Anal. 8, 1984, 381-391

[2] Agarwal R.P., Akrivis G., Boundary value problems occurring in plate deflection theory, J. Comput. Appl. Math. 8, 1982, 145-154 [

[3] Bougoffa L., Note on the explicit solutions for a boundary value problem of a secondorder nonlinear equation, Appl. Math. Lett. 21 (3), 2008, 275-278

[4] Boyd J.P., Chebyshev polynomial expansions for simultaneous approximation of two branches of a function with application to the one-dimensional Bratu equation, Applied Mathematics and Computation 142, 2003, 189-200

[5] Boyd J.P., One-point pseudospectral collocation for the one-dimensional Bratu equation, Applied Mathematics and Computation 217, 2011, 5553-5565

[6] Goyal V., On a second nonlinear boundary value problem, Appl. Math. Lett. 19 (12), 2006, 1406-1408

[7] He J.H., Variational iteration method for delay differential equations, Commun. Non-linear Sci. Numer. Simulat. 2 (4), 1997, 235-236

[8] He J.H., Variational iteration method for autonomous ordinary differential systems, Appl. Math. Comput. 114, 2000, 115-123

[9] Jalilian R., Non-polynomial spline method for solving Bratu's problem, Computer Physics Communications, Volume 181, Issue 11, 2010, 1868-1872

[10] Khuri S.A., A new approach to Bratu's problem, Applied Mathematics and Compu- tation, 147(1), 2004, 131-136

[11] Liu H.M., Variational approach to nonlinear electrochemical system, Int. J. Nonlinear Sci. Numer. Simulat. 5 (1), 2004, 95-96

[12] Liu H.M., Generalized variational principles for ion acoustic plasma waves by He's semi-inverse method, Chaos Solitons \& Fractals 23 (2), 2005, 573-576 
[13] Misir A., Tiryaki A., More on the explicit solutions for a second-order nonlinear boundary value problem, Applied Mathematics Letters 21, 2008, 1204-1208
[14] Wazwaz A.M., A reliable algorithm for obtaining positive solutions for nonlinear boundary value problems, Comput. Math. Appl. 41, 2001, 1237-1244 